\title{
La estructura de las formas libres
}

\section{The structure of free-forms}

\author{
D. Azagra(*), A. Bernabeu ${ }^{(*)}$
}

RESUMEN

La situación actual, caracterizada por un impresionante avance de las técnicas de diseño, proyecto y construcción, y por el establecimiento de una cultura digital, ha propiciado el desarrollo arquitectónico de formas que podríamos calificar como libres. El artículo valora el diverso desarrollo de estas formas libres, y el papel de la estructura en dicho proceso.

Se consideran para ello cuatro planteamientos con los que afrontar el desarrollo de estas formas libres, en función de su proceso de generación y de la influencia de la estructura: formas escultóricas, formas de conjunción estructural, formas de generación estructural o algorítmica, y formas paramétricas.

A partir de este análisis se proponen cuatro puntos de discusión: el proceso de desarrollo de las formas libres; cómo afrontar la multiplicidad de factores actuales; la respuesta y relevancia de la estructura; y el potencial de la arquitectura digital y el papel de la estructura en su desarrollo.

405-6

Palabras clave: Estructura; Arquitectura; Formas libres; Geometría compleja; Arquitectura digital.

\section{SUMMARY}

The current situation, characterised by an impressive advance of conceptual, design and construction techniques, together with an establishment of a digital culture, has led to the architectural development of the so called free-forms. This paper evaluates the diverse development of different types of free-forms, and the role of the structure in each case.

Four conceptual approaches are considered to tackle the design of free-forms as a function of both the process of generation and the influence of the structure in its development: sculptural forms, forms of concurrent structural adaptation, forms of structural algorithmic generation, and parametric forms.

Starting from this analysis, four issues are presented for discussion: the process of development of free-foms, the design approach considering the multiplicity of factors in current projects, the response and relevance of the structure in freeform architecture, and finally, the potential of digital architecture and the role of the structure in its development.
Keywords: Structure; Architecture; Free-forms: Complex geometry; Digital architecture.

(*) Oxford Brookes University. Oxford, (Reino Unido) 


\section{INTRODUCCIÓN}

Actualmente, el intenso desarrollo tecnológico que se ha producido en las técnicas auxiliares de diseño, análisis y construcción, ha generado una situación arquitectónica sin precedentes, en la que prácticamente cualquier planteamiento formal puede ser resuelto y construido. En este contexto, superados en gran medida los requisitos estáticos (de gran complejidad analítica y resolutiva en muchos casos, pero cada vez menos condicionantes del proyecto), resulta necesario analizar y reconsiderar la función de la estructura y su relación con la arquitectura.

Como punto de partida, la enorme libertad arquitectónica actual, unida a la creciente demanda social de formas novedosas y espectaculares, ha dado como resultado una gran heterogeneidad de formas y estilos, cobrando gran protagonismo las que podríamos denominar como formas libres.

Sin embargo, esta denominación de formas libres es ambigua, y puede abarcar, como veremos, planteamientos arquitectónicos diversos e incluso contrapuestos. En efecto, dentro de las formas libres podemos incluir las que son libremente trazadas por el arquitecto, aquellas cuya definición es independiente de criterios que no sean puramente formales, las que son generadas aleatoriamente, las de geometría compleja..

El presente artículo analiza y valora la respuesta que puede ofrecer la estructura a esta arquitectura de formas libres; respuesta que depende en gran medida de la interpretación que se haga de la forma libre en sí misma, y en particular de su proceso de generación o desarrollo.

\section{SITUACIÓN ACTUAL}

A la hora de considerar el desarrollo de las formas libres y la posible respuesta de la estructura, resulta necesario en primer lugar valorar brevemente el contexto arquitectónico, cultural y social en el que se producen. En este sentido se destacan a continuación tres factores fundamentales que definen y caracterizan la situación actual, y que tienen una influencia directa en el desarrollo formal considerado:

- Libertad formal sin precedentes, consecuencia del desarrollo tecnológico y técnico de los sistemas auxiliares de diseño, análisis y construcción.

- Enorme incremento de los factores y parámetros que intervienen en el proyecto.

- Establecimiento de una cultura digital, que introduce un nuevo conjunto de requisitos, parámetros y motivaciones a los que hacer frente desde la arquitectura, compatibilizando lo virtual con lo tectónico.
En primer lugar se ha producido desde finales del siglo XX un intenso desarrollo tecnológico y técnico que, en su aplicación en varios niveles en el campo de la construcción, ha modificado irreversiblemente tanto el alcance y las posibilidades técnicas y constructivas, como la propia manera de diseñar y proyectar.

Por una parte se ha producido un impresionante desarrollo de los sistemas computerizados de representación, cálculo, fabricación y montaje, que han convertido al ordenador en un potentísimo asistente en la concepción, el análisis y la construcción de propuestas altamente complejas.

Así mismo, tanto el desarrollo de potentes sistemas de cálculo (que permiten resolver con rapidez y precisión problemas de gran dificultad analítica), como la profundización actual del entendimiento estructural, han creado una situación de gran dominio del hecho estructural, permitiendo abordar con seguridad sistemas y estructuras de gran complejidad, no planteables o resolubles hasta hace relativamente poco tiempo (Figura 1).

Por último, cabe destacar la notable mejora de las propiedades y de las características de los materiales estructurales existentes, fundamentalmente en términos de calidad, resistencia, durabilidad, control y condiciones de puesta en obra, que ha contribuido a ampliar sus posibilidades técnicas y constructivas.

La conjunción de estos factores técnicos ha generado un control de las estructuras sin precedentes, propiciando una situación en la que prácticamente cualquier planteamiento formal puede ser resuelto y construido. Por primera vez en la historia de la construcción, los condicionantes estructurales y constructivos han pasado a un segundo nivel, dejando de ser limitativos y siendo posible por lo tanto obviarlos, al menos parcialmente, en el planteamiento del proyecto.

A esta situación se contrapone además el hecho de que no han aparecido recientemente materiales o sistemas estructurales que sean capaces por sí mismo de sugerir $u$ orientar nuevos planteamientos formales, de manera que la estructura ha perdido en gran medida los argumentos que le permitieron en épocas anteriores guiar y orientar el desarrollo de nuevas formas arquitectónicas (1).

Paralelamente se ha producido una insistente demanda social de formas novedosas y sorprendentes que, unida al indicado control técnico actual, ha propiciado el desarrollo y la convivencia de una gran heterogeneidad de formas y estilos, con gran predominancia de las que podríamos denominar con carácter general como formas libres. 


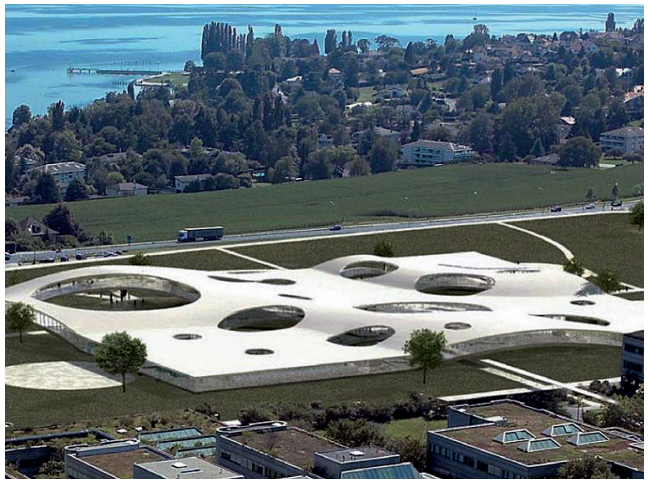

En segundo lugar, el progresivo aumento del conocimiento en diversos ámbitos, así como su consecuente división y especialización, ha generado un enorme incremento de los factores y parámetros que intervienen en la definición de un proyecto, así como una mayor sofisticación de los mismos.

La lista de requisitos y factores a los que el proyecto debe hacer frente es cada vez más larga, considerando tanto los condicionantes estructurales y constructivos, como los relativos al control energético y a los criterios medioambientales y de sostenibilidad, así como aquellos derivados del propio funcionamiento y uso del edificio (acústicos, de iluminación, de flujo de personas y vehículos...). Cabría añadir además a esta lista de requisitos o criterios técnicos, los factores económicos, por una parte, y los formales, estéticos y de comunicación o representación del edificio, por otra. Estos factores económicos y formales alcanzan en algunos casos grandes niveles de sofisticación, al vincularse junto con el desarrollo del proyecto parámetros derivados de su explotación o representación social (consideración económica conjunta de la construcción y concesión de explotación del edificio, repercusión mediática del edificio, con el consiguiente efecto publicitario).

Todos estos factores intervienen en el proyecto y deben por lo tanto ser tenidos cuenta, en mayor o menor medida, siendo así mismo susceptibles de sugerir su desarrollo en un sentido u otro, en función de la preponderancia o relevancia que se les otorgue en cada caso.

Esta multiplicidad de factores aumenta así por una parte el abanico de enfoques posibles con los que abordar el planteamiento de un proyecto, y condiciona por otra su proceso de diseño y desarrollo, como queda patente en el importante número de consultores que participan en determinados proyectos, constituyendo complejos equipos multidisciplinares de laboriosa organización y coordinación.

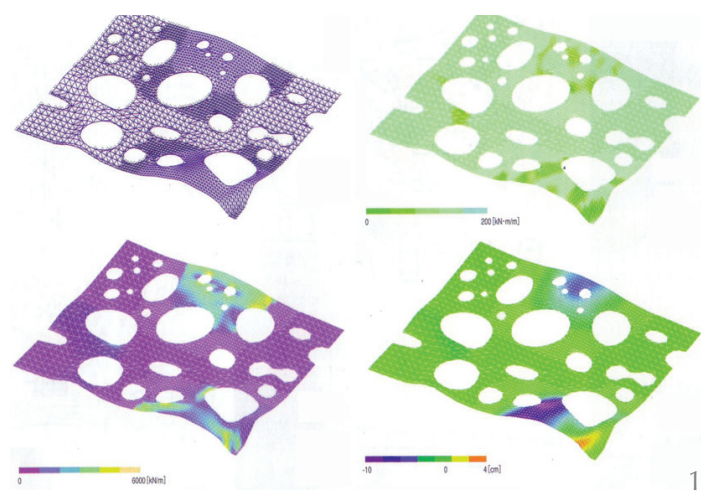

Finalmente, el reciente establecimiento de una cultura digital amenaza con transformar radicalmente el contexto social y cultural en el que se desarrolla la arquitectura, alterando los parámetros y factores que actualmente la rigen. No es el objeto del presente artículo entrar en detalle en los distintos factores que definen la actual cultura digital, aunque sí interesa destacar los siguientes aspectos, que caracterizan el contexto actual y su relación con la arquitectura (2):

- Redefinición de nuestra relación con el mundo físico, transformando el concepto de materialidad en función de las posibilidades de la realidad virtual, que abarca tanto niveles de representación y recreación o investigación espacial como de cyber-sociabilidad, y de la relación u oposición entre lo real y lo virtual (Figura 2-página siguiente).

- Importancia otorgada a las preferencias y elecciones individuales, junto con el establecimiento de una cultura y sociedad globales. La actual sociedad digital establece tanto la globalización de los productos y sistemas como las posibilidades de configurar personal e individualmente dichos productos, haciendo patente la dualidad entre lo estándar/global/colectivo y lo úni$\mathrm{co} /$ personal/individual (Figura 2).

- Relevancia de los acontecimientos y los eventos de todo tipo, de manera que lo que ocurre pasa a ser más importante que lo que físicamente existe, como pone de relevancia Internet, donde los contenidos son actualizados instantáneamente en función de los últimos acontecimientos, en un vertiginoso proceso que nos deja la extraña sensación de vivir en un presente permanente.

En su relación con la arquitectura, estos factores suponen la crisis de la tectónica tradicional, en su vinculación y dependencia al objeto y la materialidad física, reclamando el desarrollo de una nueva tectónica que sea capaz de acercar la abstracción de la cultura y sociedad digitales a la concreción que la realidad arquitectónica demanda. 


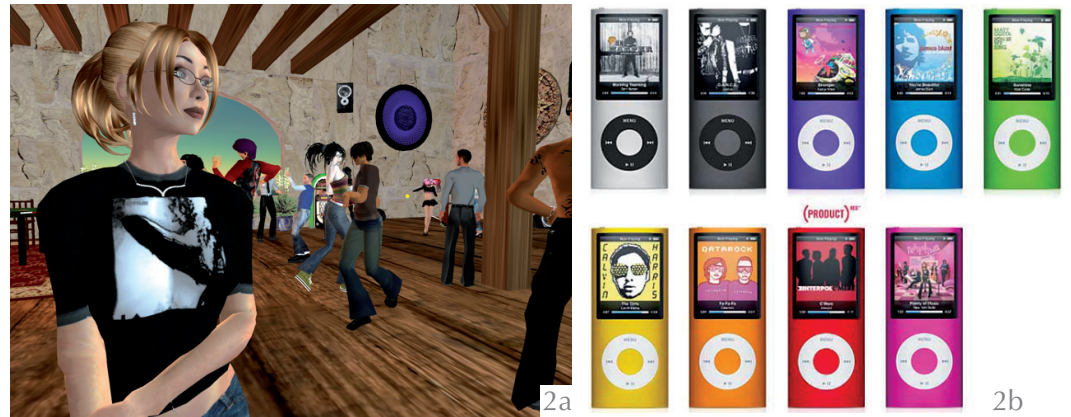

\section{LA ARQUITECTURA DE LAS FORMAS LIBRES Y LA RESPUESTA ESTRUCTURAL}

2a. Secondlife -Realidad virtual y cyber-sociabilidad-.

2b. IPod -Dualidad entre lo estándar/global y lo único/individual-.

3. Guggenheim. Bilbao, 1996. Frank Gehry. Boceto y Adaptación a Catia.

Este contexto arquitectónico y social ha transformado necesariamente la relación entre la arquitectura y su soporte resistente, haciendo necesario redefinir los criterios y conceptos que rigen la definición y el desarrollo de la estructura, y obligando a reconsiderar y repensar sus posibilidades de participación en el planteamiento formal de la arquitectura, mediante nuevas estrategias y criterios de diseño estructural (3).

Especialmente sintomático de la situación arquitectónica actual resulta la predominancia de lo que podríamos denominar arquitectura de formas libres, que aprovecha las posibilidades técnicas existentes para proponer formas y geometrías que se caracterizan en conjunto por una enorme libertad formal.

La valoración y consideración de las posibles respuestas o estrategias estructurales en este caso está necesariamente vinculada a la que se haga a nivel arquitectónico. Se propone por lo tanto en el presente artículo como proceso de análisis, la consideración de cuatro planteamientos proyectuales posibles con los que enfrentarse a la situación actual, desde el punto de vista de la libertad formal del proyecto, y de su proceso de generación y desarrollo: formas escultóricas, de adaptación o conjunción estructural, de generación estructural o algorítmica, y formas paramétricas.

\subsection{Formas escultóricas}

En primer lugar, la situación más clara de formas libres es aquella en la que las formas derivan directamente del trazo libre del arquitecto, ajenas a cualquier criterio que no sea formal, sin tener en cuenta, al menos explícitamente, consideraciones de índole constructivo o estructural.

En estos casos el arquitecto aprovecha las posibilidades que ofrecen los desarrollos técnicos y estructurales, planteando nuevas formas y geometrías que trascienden las resultantes de la aplicación directa de la lógica estructural, ofreciendo una nueva libertad formal. En este sentido podemos considerar, a modo de referente histórico, el edificio de la TWA de Eero Saarinen, que parte de los desarrollos técnicos y formales de Torroja en el campo de las láminas delgadas, de carácter estricto, para adoptar una libertad formal y una plasticidad nuevas.

Actualmente, el ejemplo más claro de estas formas libres lo constituyen sin lugar a dudas los proyectos de Frank Gehry, y en particular el museo Guggenheim de Bilbao. Frank Gehry aprovecha las posibilidades técnicas actuales en cuanto a software de representación y control de geometrías complejas, mediante la adaptación del programa de diseño aeronáutico Catia al campo de la arquitectura y la construcción, con el objetivo de reproducir fielmente las geometrías libres que propone (4).

En esta adaptación o aplicación de un programa de altas prestaciones como Catia, resulta sintomático sin embargo que su utilización se limite a la reproducción exacta de una forma ya definida, renunciando a su potencial como asistente o herramienta de diseño. $Y$ en efecto, el proceso creativo de Frank Gehry es tradicional o artesanal en este sentido, dibujando a mano, y trabajando con bocetos y maquetas físicas, que contrastan con el avanzado, exhaustivo y complejo proceso que requiere su tratamiento posterior con Catia, que queda limitado así a una función auxiliar, aunque necesaria, de precisa reproducción (Figura 3).

m
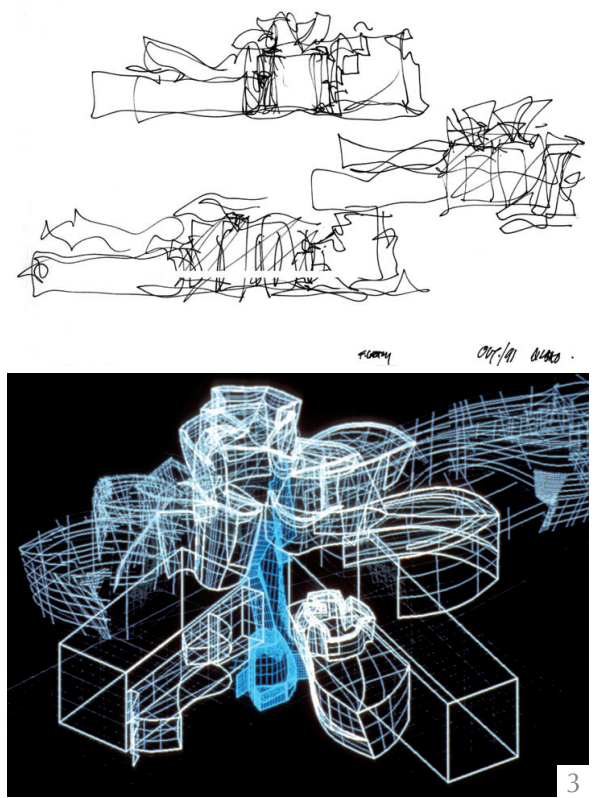

La posible respuesta de la estructura en estos casos es complicada, al enfrentarse a una forma ya completamente definida, y que le es ajena, a la cual debe adaptarse sin alterarla. Así, en el caso del museo Guggenheim la estructura resuelve eficaz y elegantemente una 
geometría de gran complejidad constituida por superficies alabeadas y curvas, utilizando celosías trianguladas espaciales que se adaptan con facilidad y flexibilidad a la geometría requerida, y contribuye a generar espacios de gran interés (5). Sin embargo, el sistema estructural se acomoda a una forma ya definida, sin contribuir a su definición (Figura 4). La estructura hace posible el proyecto, pero no influye de manera relevante en el diseño del mismo, que es obra exclusiva del arquitecto.
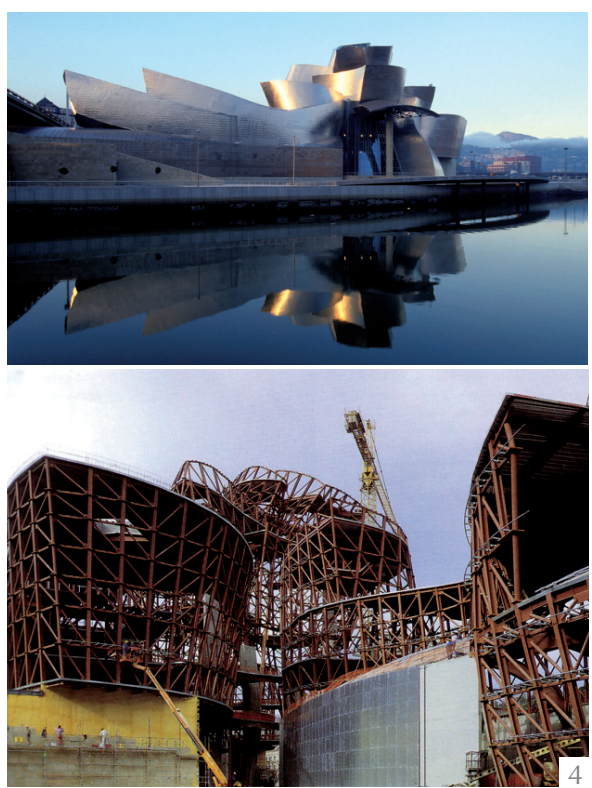

Cabe en estos casos plantearse cómo debe enfrentarse la estructura a estas formas libres, si debe limitarse a reproducir fielmente la geometría propuesta (copiar a escala la maqueta original), encajando la estructura lo mejor posible, o si puede proponer un sistema coherente con el planteamiento arquitectónico pero que modifique la forma inicial propuesta.

Esta cuestión es abordada por el ingeniero Cecil Balmond al enfrentarse a la estructura del estadio de Chemnitz, de Peter Kulka, que planteaba una cubierta de gran libertad formal, como metáfora de una nube, apoyada en una distribución aleatoria de pilares, como árboles de un bosque (Figura 5) (6). Balmond se pregunta cómo copiar una maqueta de papel y transformarla en una estructura sólida, si debe reproducirse exactamente cada curva y cada pliegue, y propone alternativamente plantear una estrategia que sea capaz de generar una tipología similar. "No copiar, sino construir en dirección a una idea, reinventándola" (7).

\subsection{Formas de adaptación o conjunción estructural}

Este planteamiento nos lleva al segundo nivel dentro del análisis de las formas libres, en el que existe un desarrollo conjunto de las mismas, que tiene en cuenta los criterios y requisitos estructurales y constructivos.

Este desarrollo conjunto de las formas libres se ve además animado por la difícil aceptación de la arbitrariedad en la arquitectura. $Y$ es que, al contrario que en otras artes, la arquitectura raramente se siente cómoda con la libertad plástica, amparándose en razones o reglas que guíen o justifiquen el desarrollo de la forma. Esta reticencia a la arbitrariedad formal explica la poco entusiasta acogida que a menudo reciben los proyectos de Gehry, uno de los pocos arquitectos contemporáneos que acepta y defiende abiertamente la arbitrariedad en su arquitectura, consciente de que "sus ordenadores harán posible, en primer lugar, la definición de sus formas caprichosas, garantizando después su construcción" (8).

Desde este punto de vista, la arquitectura, reacia a aceptar la libertad o arbitrariedad de las formas por sí mismas, buscaría sistemas o planteamientos que le permitan investigar y desarrollar determinados aspectos formales, bajo el amparo de una determinada lógica (estructural o constructiva, en este caso), que le permita presentar la forma deseada como algo inevitable, el resultado de un determinado proceso, y no como un capricho formal del arquitecto.

Así tendríamos aquellos proyectos que parten de un planteamiento formal inicial del arquitecto pero que buscan integrar los distintos condicionantes (funcionales, estructurales, constructivos, de instalaciones, acústicos...), de manera que la forma resultante sea coherente con su soporte resistente, y satisfaga así mismo el resto de requisitos, encontrando un equilibrio entre el deseo formal inicial del arquitecto y la realidad constructiva del proyecto. En estos casos se defiende que la forma, aun de gran complejidad geométrica en algunos casos, no es el resultado de un gesto arbitrario del arquitecto, sino que responde a una serie de parámetros y de lógicas, que terminan por converger en la geometría resultante. La forma no es así un a priori del proyecto, sino el resultado de un proceso.
4. Guggenheim. Bilbao, 1996. Frank Gehry. Vista exterior y Ejecución de la estructura.

5. Estadio de Chemnitz. Alemania, 2003. Peter Kulka, Cecil Balmond. Maqueta.

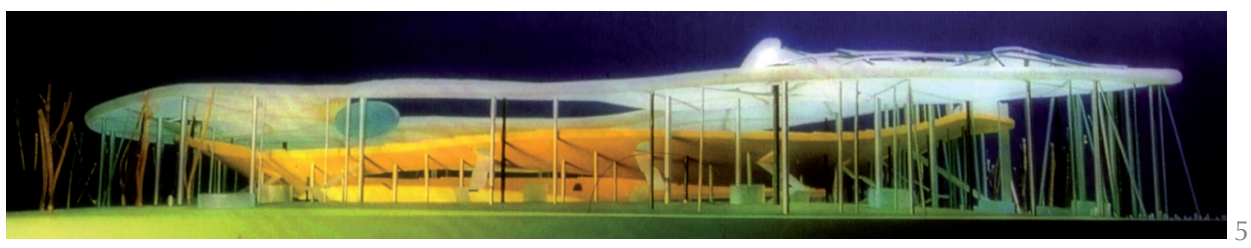


6a. Campus de la Justicia. Madrid, 2008. Foster+Partners; Buro Happold, NB35.

6b. Estructura salas de vista Audiencia Provincial. Generación salas de vista Tribunal Superior de Justicia.

7. Cubierta del British Museum. Londres, 2000. Foster+Partners, Buro Happold. Modelo de desarrollo de la malla estructural y vista interior
En este sentido se plantea el artículo de los autores "Searching for the right form", que explora el papel de la estructura en el desarrollo de formas de geometría compleja, a partir del proceso de diseño de dos proyectos de Foster+Partners para Campus de la Justicia en Madrid (Figura 6). Se plantea en estos casos cómo debe afrontar la estructura su relación con la arquitectura, y cómo debe ser el proceso de diseño y desarrollo de formas de geometría libre o compleja, si existe una única geometría común o dos geometrías distintas, una arquitectónica y otra estructural, y quién debe definir esa geometría, el arquitecto o el ingeniero (9).
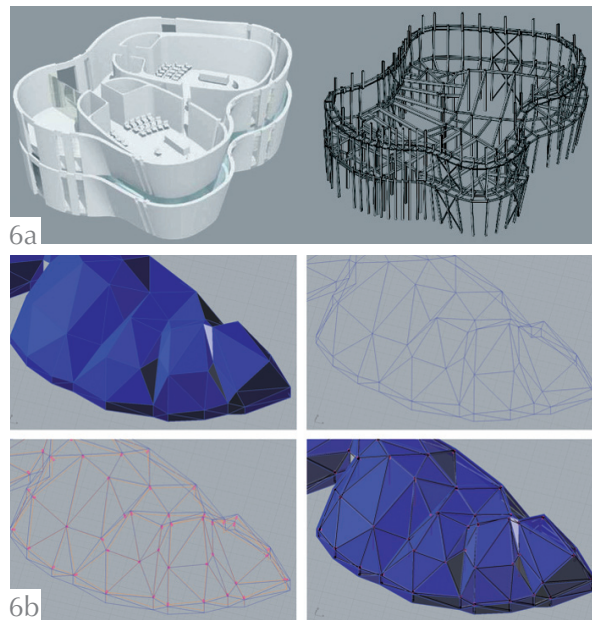

La geometría aparece así en estos casos como el punto de conexión, de relación y diálogo entre la arquitectura y la estructura. En algunos casos la estructura se deberá acomodar a una determinada geometría, aunque dotándole siempre de una cierta lógica constructiva o estructural, mientras que en otros será la encargada de liderar el proceso de desarrollo de la forma, a partir de sus propios mecanismos y procesos.

\subsection{Formas de generación estructural o algorítmica}

Dentro de este nivel de definición conjunto de las formas libres podríamos considerar también los proyectos que utilizan para el planteamiento o desarrollo de la forma sistemas basados en la optimización de la estructura.
Así, la cubierta del British Museum es el resultado de un sistema de desarrollo de la forma que parte de la geometría que adoptaría una película de jabón sometida a los mismos condicionantes geométricos que la cubierta (perímetro exterior rectangular e interior circular, altura total disponible muy ajustada), pero modificándola y controlándola mediante modelos computerizados, a fin de optimizar su comportamiento (10 y 11). El resultado es una malla estructural que se adapta de forma natural a las geometrías de los perímetros rectangular exterior y circular interior, a la vez que controla el nivel de tensiones en las distintas zonas, lo que permite el empleo de elementos estructurales metálicos de pequeño tamaño, garantizando la transparencia deseada (Figura 7).

En esta misma línea, el ingeniero japonés Mutsuro Sasaki, colaborador habitual de Toyo Ito y de Arata Isozaki, ha desarrollado dos sistemas de generación de la forma, basados respectivamente en la optimización de su comportamiento para el desarrollo de cáscaras de hormigón de curvatura libre y para el planteamiento de estructuras y formas arbóreas. Su propuesta de optimización computerizada de superficies de hormigón de curvatura libre se puede englobar en la tradición de las cáscaras de hormigón y las láminas delgadas, como continuación del trabajo de Eduardo Torroja, Felix Candela o Heinz Isler, mientras que para la generación de estructuras ramificadas o arbóreas utiliza un sistema de optimización evolutiva de la forma que permite, a partir de un proceso iterativo, generar estructuras que evolucionan hacia un estado tensional uniforme, que optimiza la respuesta estructural en función de unas condiciones de contorno determinadas (12).

Sin embargo, las formas resultantes en estos casos difícilmente se pueden calificar como óptimas, debiendo entenderse estos procesos más como una herramienta de diseño basada en el comportamiento de la estructura, que como un proceso de definición de formas estructuralmente óptimas. Así, la estructura arbórea del Centro de Convenciones de Doha (de Arata Isozaki y optimizada por el grupo SMART de Buro
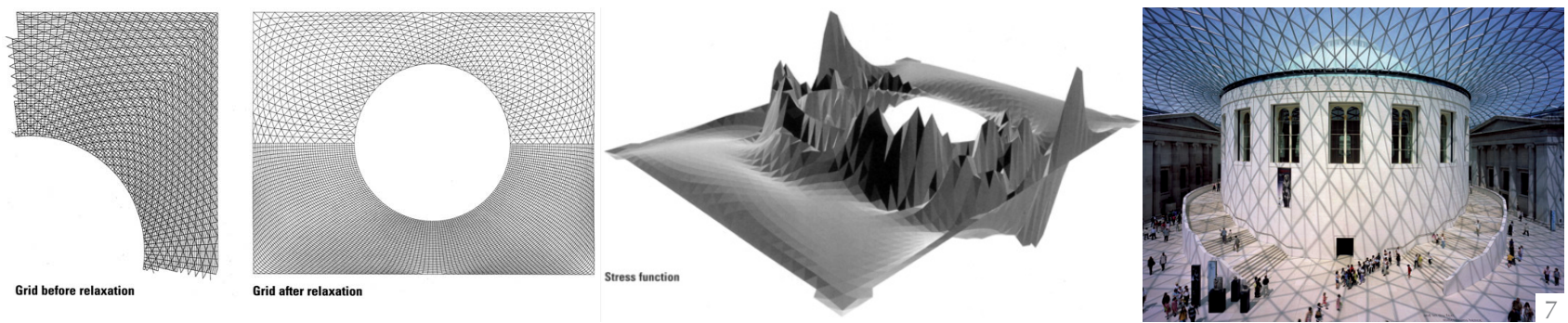

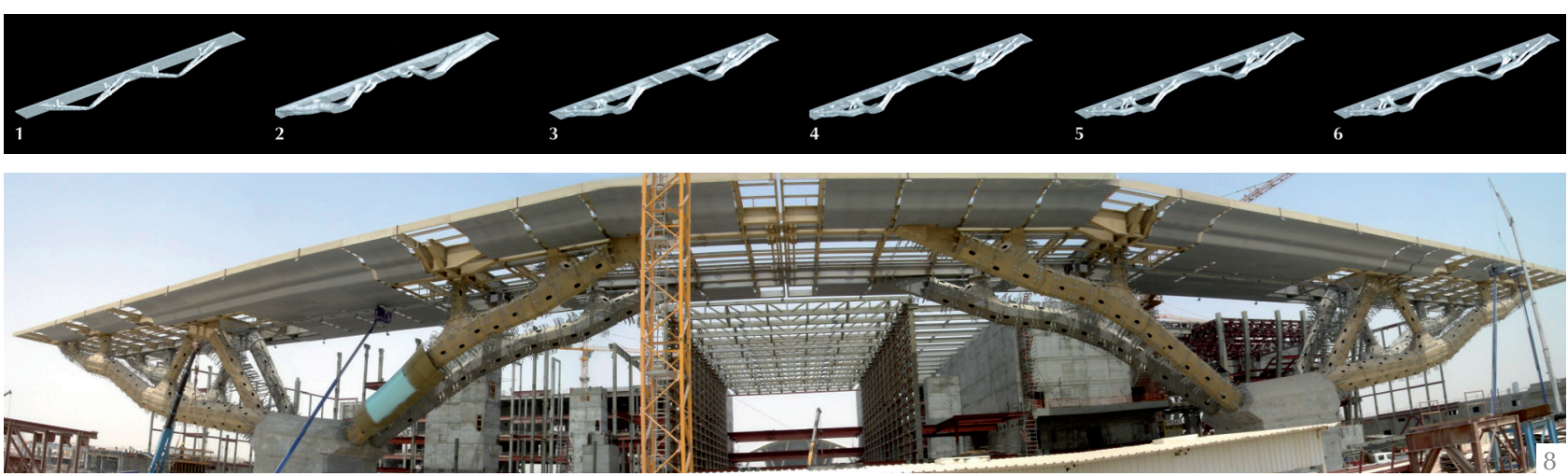

Happold) dista mucho de ser una forma óptima, si bien es cierto que optimiza su comportamiento una vez establecidos una serie de condicionantes de partida y de criterios sobre su desarrollo (Figura 8). Así mismo, la aplicación de estos sistemas a menudo derivan en formas de gran complejidad y libertad geométrica, difíciles y laboriosas de ejecutar, poniendo en cuestión su proceso de construcción la autenticidad de su deseada optimización. Ofrecen sin embargo un interesante sistema de desarrollo de geometrías complejas o libres, en el que la forma y su comportamiento resistente se relacionan e identifican inequívocamente.

Dentro de este proceso de desarrollo de formas libres de manera controlada, a partir de determinados procesos, el potencial que el control computacional de geometrías complejas ofrece (capaz de generar infinitud de variantes posibles mediante la modificación específica de algunos de los parámetros que la definen), ha propiciado el desarrollo de formas que, si bien presentan una enorme complejidad geométrica, permiten en su proceso generador dar cabida a otros criterios y factores del proyecto, más allá de los estrictamente formales, constructivos o estructurales. En este sentido se puede valorar el trabajo de arquitectos como Aranda y Lasch, o los desarrollos geométricos y estructurales del Departamento de Geometría Avanzada de Ove Arup \& Partners (AGU).

Así, en el proyecto del pabellón temporal de la Serpentine Gallery de 2002 en Londres, de Toyo Ito y Cecil Balmond, el objetivo era definir el patrón de una piel estructural de gran libertad, que se extendiera por la fachadas y cubierta, de manera que no se percibiera como un elemento estructural. Para ello, alternativamente a la opción de trazar la malla libremente, se optó por utilizar un algoritmo geométrico basado en un cuadrado que crece conforme gira, y del que se prolongan sus lados de manera que se crucen entre sí, extendiéndose por toda la superficie de la cubierta y las fachadas (13). El resultado es una compleja malla estructural de líneas que se cruzan entre sí, aparen- temente de forma aleatoria, y en la que se percibe de alguna manera el dinamismo y la relación geométrica de su origen (Figura 9).
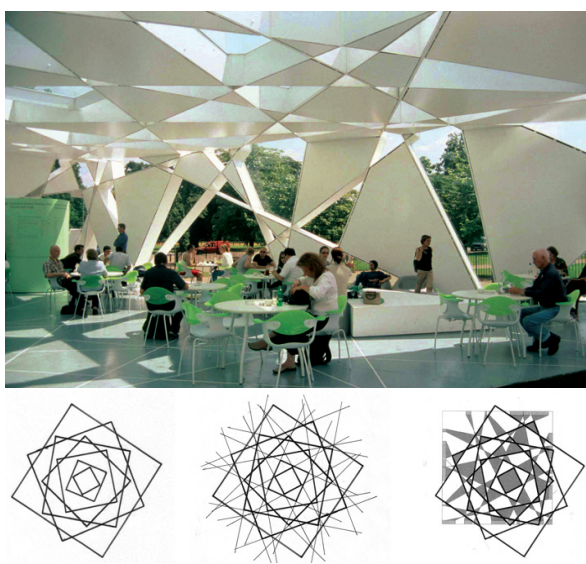

El proyecto grotto, realizado por Aranda y Lasch en colaboración con el AGU, constituye también un ejemplo interesante de esta aproximación algorítmica al desarrollo de formas libres. El objetivo en este caso era definir un sistema estructural tridimensional de gran libertad, que permitiera múltiples combinaciones, tomando como referente formal la imagen de una gruta. Para ello, se desarroIló un sistema modular basado en dos procesos geométricos y matemáticos (las series de Danzer y los diagramas de Voronoi), que dan como resultado cuatro poliedros que se pueden combinar entre sí de multitud de formas posibles, generando un sistema tridimensional autoportante que, a pesar de su aparente aleatoriedad presenta una lógica modular, aunando libertad formal y eficiencia constructiva (Figura 10) (14).

También con idea de permitir diversas configuraciones se plantea el prototipo de cubierta propuesto por Kristina Shea. El sistema consiste en una serie de módulos triangulares que se definen y conectan entre sí en función de los condicionantes geométricos específicos del emplazamiento en el que se sitúa, de manera que su forma es el resultado controlado de un proceso que depende de una serie de parámetros y factores geométricos de inicio (15) (Figura 11).
8. Centro de convenciones de Doha, Quatar, 2011. Arata Isozaki, Mutsuro Sasaki, Buro Happold. Sistema de Optimización Evolutiva de la Estructura y Vista de la construcción.

9. Pabellón temporal Serpentine Gallery. Londres, 2002. Toyo Ito, Cecil Balmond. Generación de la geométrica de la malla estructural y vista interior. 
10. Proyecto Grotto. 2004. Aranda\&Lasch, AGU Arup. Vista general y Proceso de generación de los módulos base.

11. Prototipo sistema triangular de cobertura, 2002. Kristina Shea. Desarrollo geométrico y Estado final.

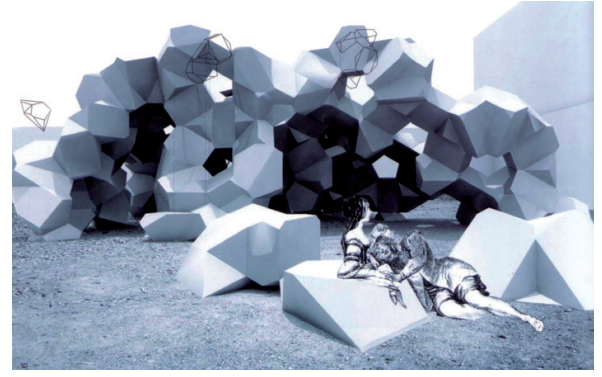

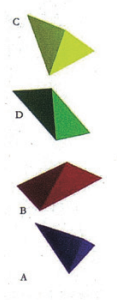

Tiles

10

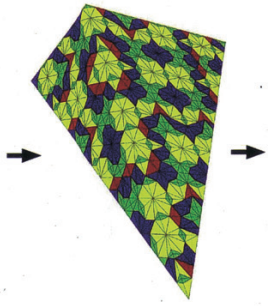

Danzer 3D tiling

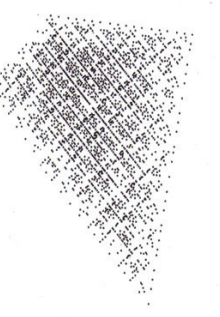

Cloud of points

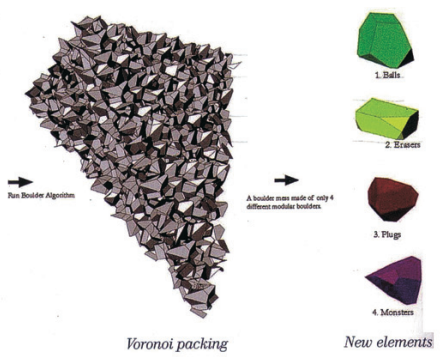

New elements
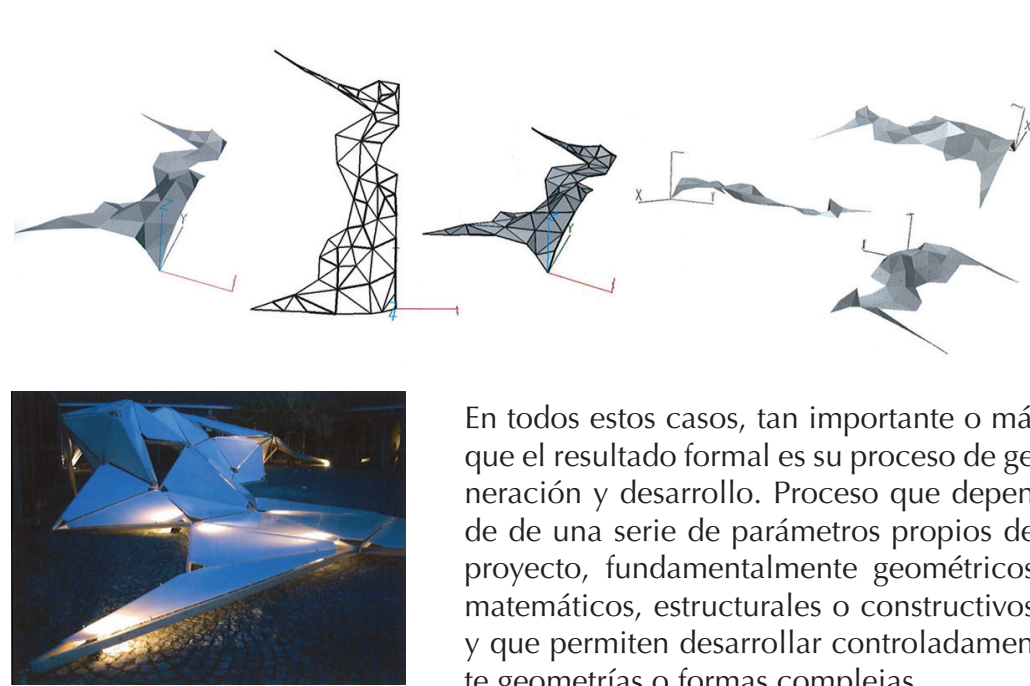

11

12a. Arquitectura digital. Softoffice, 2000. NOX.

12b. Mercedes Benz Museum. Sttutgar (Alemania), 2006. UN Studio, Werner Sobek.
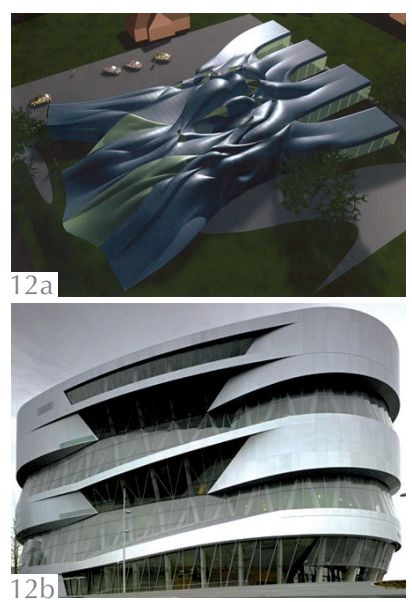

En todos estos casos, tan importante o más que el resultado formal es su proceso de generación y desarrollo. Proceso que depende de una serie de parámetros propios del proyecto, fundamentalmente geométricos, matemáticos, estructurales o constructivos, y que permiten desarrollar controladamente geometrías o formas complejas.

Se pueden entender o interpretar también estos sistemas como argumento o justificación para el planteamiento de formas libres, tratando de rechazar su arbitrariedad amparados en el proceso algorítmico que las genera, bien que en algunos casos este proceso no sea sino un "juego" matemático o geométrico, igualmente arbitrario.

\subsection{Formas paramétricas}

Finalmente, en este proceso de desarrollo de la arquitectura vinculado a los nuevos desarrollos tecnológicos y computacionales, el establecimiento de una cultura digital en la sociedad actual ha propiciado la consideración de procesos generadores de lo que podríamos denominar arquitectura digital, que tratan de integrar en el proceso de desarrollo del proyecto toda una serie de factores y complejidades, vinculadas no sólo al proyecto en sí mismo o a las técnicas constructivas y arquitectónicas, sino en un sentido más global a todo tipo de motivaciones y parámetros económicos, sociológicos o culturales.
Los proyectos de arquitectos como Greg Lynn, Ben van Berkel o muy especialmente Zaha Hadid defienden esta aproximación a la arquitectura, que Patrik Schumacher, de Zaha Hadid Architects, ha definido como Parametricismo, justificándola como el nuevo gran estilo arquitectónico después del Modernismo (Figura 12) (16).

De acuerdo con el planteamiento paramétrico que propone Shumacher, el proceso parte de una serie de principios, que organiza en negativos o tabús (evitar formas rígidas -ausencia de maleabilidad-, evitar la repetición -ausencia de variedad-, evitar la mezcla de elementos aislados inconexos -ausencia de orden-...), y positivos o dogmas (las formas deben ser blandas, los sistemas deben ser diferenciados -gradientes- e interdependientes -correlaciones-...). A partir de estos principios se organiza el sistema de definición y desarrollo del proyecto, en el que los distintos parámetros son fácilmente modificables, pudiendo orientar el resultado en función de los intereses arquitectónicos o formales, hasta alcanzar la expresividad deseada.

En estos casos, la arquitectura entendida como el resultado de un conjunto de parámetros o factores diversos que, combinados, diferenciados y correlacionados entre sí, definen un desarrollo formal maleable, plantea la cuestión sobre el papel que puede jugar la estructura, si los requisitos estructurales son un parámetro más a introducir en la ecuación, o si por el contrario pueden participar activamente en definir los patrones que rigen y controlan el desarrollo paramétrico del proyecto.

Así, se produce en estos proyectos a menudo una importante desvinculación entre la forma arquitectónica y su planteamiento estructural (expresión arquitectónica y tectónica), como pone de manifiesto por ejemplo el Phaeno Science Center en Alemania, de Zaha Hadid, cuya apariencia exterior de hormigón resulta contradictoria con la importancia de la estructura metálica interior (Figura 13).

Probablemente sea pronto todavía para valorar en profundidad el resultado proyectual de estos planteamientos y su influencia en el desarrollo de la arquitectura futura. No obstante, en la mayoría de los casos, carecen de momento en su aplicación práctica de la coherencia y profundidad que cabría esperar de sus consideraciones teóricas. Excesivamente preocupados por los aspectos formales, acordes con la imaginería visual digital actual (17), evitan afrontar en profundidad los complejos retos que propone la arquitectura digital, como vínculo y catalizador de la sociedad y cultura digitales con la realidad tectónica de la arquitectura. 


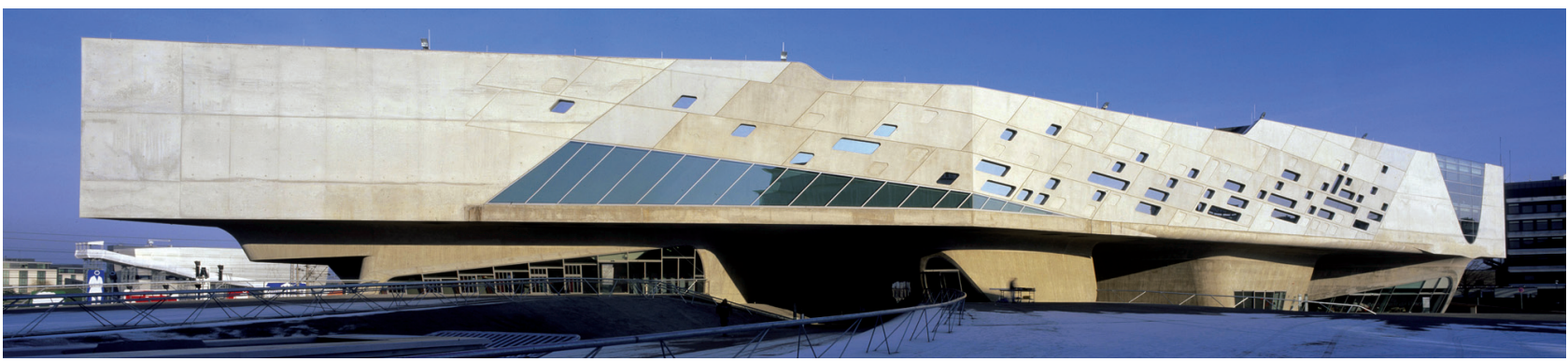

\section{DISCUSIÓN}

A partir del análisis expuesto planteamos a continuación cuatro puntos de reflexión y discusión: sobre el proceso de desarrollo de las denominadas formas libres; sobre cómo debe enfrentarse el proyecto a la multiplicidad de factores actuales; sobre la respuesta y la relevancia de la estructura en esta arquitectura de formas libres; $y$ sobre el potencial de la arquitectura digital y el papel que puede jugar la estructura en su desarrollo.

\section{Sobre el proceso de desarrollo} de las denominadas formas libres

En primer lugar, el proceso de desarrollo de las denominadas formas libres puede ser, como hemos visto, muy variable, dependiendo de las motivaciones o consideraciones que se tengan en cuenta. En consecuencia, el nivel de relevancia de la estructura (y de manera similar de otros requisitos y factores del proyecto), dependerá directamente de la actitud con la que se aborde su desarrollo formal, y que, en este caso, se puede considerar en varios niveles:

- El entendimiento de que el proyecto se debe abordar desde una conciencia clara de los distintos requisitos y parámetros que intervienen en el mismo, conscientes de la realidad tectónica y constructiva de la arquitectura.

- El deseo de justificar el desarrollo de formas de gran libertad geométrica, de manera que éstas se vean no como el deseo arbitrario o caprichoso del arquitecto, sino como el resultado de un determinado proceso, basado en cuestiones técnicas o constructivas. - El reconocimiento de los distintos requisitos del proyecto no como condicionantes molestos que hay que controlar, evitando que interfieran o desvirtúen en su planteamiento formal, sino como verdaderas herramientas de diseño, ofreciendo nuevas posibilidades proyectuales.

\section{Sobre la multiplicidad de factores en el proyecto}

Por otra parte, al enfrentarse el proyecto a un número creciente de factores y parámetros de distinta índole, existe la tentación de tratar de plantear una solución equilibrada, que responda uniformemente a los distintos requisitos que integran el proyecto, Ilevando cada uno hasta el punto en el que comenzaría a interferir con otros criterios o factores, de manera que queden todos razonablemente satisfechos.

Sin embargo, tratar de satisfacer todos los requisitos a los que hace frente un proyecto, respondiendo positivamente a todas las sugerencias y motivaciones derivadas de éstos, puede dar lugar a soluciones de compromiso: correctas, adecuadas o incluso eficientes en cierto sentido, pero carentes de fuerza. Al tratar de ofrecer una respuesta coherente y equilibrada de todos los factores, se corre el riesgo de no satisfacer plenamente ninguno, de que el resultado sea excesivamente metódico, neutro, imparcial y desapasionado.

Pueden resultar así más interesantes propuestas más arriesgadas, que potencien decididamente un determinado factor como verdadero argumento generador del proyecto, aun subordinando hasta cierto punto otros requisitos o intereses posibles. Un proyecto no es una lista de condicionantes y necesidades a cumplir (funcionalidad, relación e integración con el entorno, estabilidad estructural, eficiencia energética, contención económica...). Un proyecto exige pronunciarse, adoptar una postura firme y precisa, tomar un camino en detrimento de otros posibles.

Así, aplicar decididamente una lógica determinada a un proyecto no es casi en ningún caso una necesidad (o no es únicamente una necesidad), ni constituye una solución óptima que ofrezca ventajas objetivas invariables. Es, al contrario, una opción de proyecto, una herramienta de diseño capaz de ofrecer un argumento para su desarrollo formal.

Sobre la respuesta y la relevancia de la estructura en las formas libres

A partir de las reflexiones anteriores se puede abordar la cuestión de cuál debería ser la actitud de la estructura ante la libertad arquitectónica actual, si debería potenciarla, favoreciendo el desarrollo de formas libres, exentas de cualquier restricción téc-

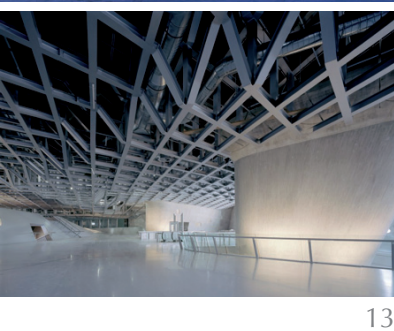

13. Phaeno Science Center. Wolfsburg (Alemania), 2005. Zaha Hadid, Adams Kara Taylor. Vista exterior de hormigón y Estructura metálica interior. 
nica, o por el contrario restringirla, tratando de evitar la desvinculación entre la forma y su soporte resistente.

La respuesta a esta cuestión entendemos que no puede ser única, de manera que la relevancia de la estructura en esta arquitectura de formas libres puede ser muy diversa, como queda de manifiesto en los distintos casos identificados:

- En las formas escultóricas la estructura carece de relevancia a nivel de diseño, aparece a posteriori, resuelve el proyecto pero no participa en su definición.

- En las formas de adaptación o conjunción estructural, la estructura ofrece resultados y respuestas a lo largo del proceso de diseño, orientando y participando en su desarrollo. - En las formas de generación estructural o algorítmica son los propios criterios geométricos o de optimización y eficiencia estructural los que originan y dan razón a la forma. - Finalmente, en las formas paramétricas la relevancia de la estructura depende de la importancia y expresividad en relación con el resto de parámetros que participan en el proceso.

En un extremo se supone así la posibilidad de encontrar una respuesta estructural a cualquier forma, sin necesidad de que la lógica estructural importe. En el otro extremo se asume la existencia de una forma estructuralmente óptima que puede ser ge- nerada a través de determinados procesos o algoritmos, sin necesidad de que exista una forma conceptual previa. Y cualquiera de estos dos planteamientos proyectuales puede ser a priori perfectamente válido.

Sobre la arquitectura digital y el papel de la estructura

El establecimiento de una cultura digital aumenta el grado de complejidad del problema, al abrir un nuevo campo de aplicación a la arquitectura, con enorme potencial social, y que no está todavía precisamente enunciado ni menos aún resuelto: el compatibilizar desde la arquitectura las necesidades y motivaciones virtuales de la nueva sociedad digital con la realidad física y funcional que toda construcción demanda.

En este contexto, ¿debe la estructura desempeñar el papel de orientar la libertad formal guiando su desarrollo con mayor o menor protagonismo, de acuerdo con el tipo de posicionamiento arquitectónico respecto a ella (su expresión o negación, la aceptación de sus principios o la represión de su realidad)?

O más aún, ¿es la estructura el elemento esencial para conectar la arquitectura digital con la realidad tectónica y constructiva, capaz de otorgar una decisiva fuerza de expresión y coherencia a las formas libres?

\section{BIBLIOGRAFÍA}

(1) Manterola, J.: "La estructura resistente en la arquitectura actual". Informes de la construcción, vol. 50, n. ${ }^{\circ}$ 456-457, julio/agosto-septiembre/octubre, 1998, pp. 15-45.

(2) Picon, A.: Digital culture in architecture. Birckhäuser, Basel, 2010.

(3) Bernabeu Larena, A.: Estrategias de diseño estructural en la arquitectura contemporánea. El trabajo de Cecil Balmond. Tesis Doctoral dirigida por Ricardo Aroca Hernández-Ros. Escuela Técnica Superior de Arquitectura de Madrid, UPM. 2008. http://oa.upm.es/910/.

(4) Steele, J.: Arquitecture y revolución digital. Gustavo Gili. Barcelona, 2001.

(5) Manterola, J.: "La estructura resistente en la arquitectura actual (continuación)". Informes de la construcción, vol. 57, n. ${ }^{\circ}$ 499-500, septiembre/octubre-noviembre/diciembre, 2005, pp. 9-35.

(6) Kulka, P. y Königs, U.: Sportstadium Chemnitz 2002. Aedes east. Berlín, 1996.

(7) Balmond, C.: Informal. Prestel Verlag, 2002.

(8) Moneo, R.: Sobre el concepto de arbitrariedad en arquitectura. Discurso leído en el acto de recepción pública de académico electo. Real Academia de Bellas Artes de San Fernando, 16 enero 2005.

(9) Bernabeu Larena, A. y Azagra, D.: "Searching for the right form-The role of structural engineers in the design of two complex buildings in Madrid". The structural engineer, 88 (13) 6 July 2010, pp. 28-34.

(10) Cook, M.: "Digital Tectonics. Historical Perspective-Future Prospect". En Leach, N.; Turnbull, D. y Williams, C.: Digital tectonics. Wiley-Academy. Gran Bretaña, 2004, pp. 40-49.

(11) Williams, C.: "Design by algorithm". En Leach, N.; Turnbull, D. y Williams, C.: Digital tectonics. Wiley-Academy. Gran Bretaña, 2004, pp. 79-85.

(12) Sasaki, M.: Flux structure. Toto Shuppan, Tokio, 2005.

(13) $\mathrm{a}+\mathrm{u}$ (ed.): "Cecil Balmond". a + u. Architecture and Urbanism, 2006.

(14) Aranda, B. y Lasch, C.: Tooling. Pamphlet architecture, n. ${ }^{\circ} 27$. Princeton architectural press, New York, 2006.

(15) Shea, K.: "Directed randomness". En Leach, N.; Turnbull, D. y Williams, C.: Digital tectonics. Wiley-Academy. Gran Bretaña, 2004, pp. 89-101.

(16) Schumacher, P.: "Let the style war begin". Architect's Journal, 06 May 2010, pp. 41-45.

(17) Krauel, J.: "Arquitectura digital". Innovación y diseño. Links. 2010. 